\title{
Periodontal health, perceived oral health, and dental care utilization of breast cancer survivors
}

\author{
L. Susan Taichman, MS, MPH, PhD'; Jennifer J. Griggs, MD, MPH ${ }^{2,3}$; Marita R. Inglehart, Dr. phil. habil. ${ }^{1,4}$ \\ 1 Department of Periodontics and Oral Medicine, University of Michigan School of Dentistry, Ann Arbor, MI, USA \\ 2 Department of Internal Medicine, Hematology and Oncology Division, University of Michigan, Ann Arbor, MI, USA \\ 3 Department of Health Management Policy, School of Public Health, Ann Arbor, MI, USA \\ 4 Department of Psychology, College of Literature, Science and Arts (LS\&A), University of Michigan, Ann Arbor, MI, USA
}

Keywords

breast neoplasms; quality of life; postmenopause; women; dental health services; NHANES; survivorship; oral health-related quality of life.

\section{Correspondence}

Dr. Susan Taichman, Department of Periodontics and Oral Medicine, University of Michigan School of Dentistry, 1011 North University Ave., Ann Arbor, Ml 48109-1078. Tel.: (734) 763-8073; Fax: (734) 763-5503; e-mail: hipolite@umich.edu. Susan Taichman is with the Department of Periodontics and Oral Medicine, University of Michigan School of Dentistry. Jennifer J. Griggs is with the Department of Internal Medicine, Hematology and Oncology Division, University of Michigan and Department of Health Management Policy, School of Public Health. Marita R. Inglehart is with the Department of Periodontics and Oral Medicine, University of Michigan School of Dentistry and Department of Psychology, College of Literature, Science and Arts (LS\&A), University of Michigan.

Received: 2/7/2014; accepted: 12/19/2014.

doi: 10.1111/jphd.12084

Journal of Public Health Dentistry 75 (2015) 148-156

\begin{abstract}
Objectives: This population-based analysis examined the prevalence of periodontal diseases along with the self-perceived oral health and patterns of dental care utilization of breast cancer survivors in the United States.

Methods: Data from the 1999-2004 National Health and Nutrition Surveys were utilized, examining information from 3,354 women between 50 and 85 years of age. Primary outcomes were gingivitis and periodontitis, self-perceived oral health, and dental care utilization. Logistic regression analyses were used to estimate relationships of breast cancer diagnosis and primary outcomes while controlling for confounding factors.

Results: Breast cancer survivors were more likely to be older than 55 years, white, nonsmokers, have higher levels of education and income, and a higher prevalence of osteoporosis. Breast cancer survivors were significantly less likely to have dental insurance $(P=0.04)$. Utilization of dental services and reason for last dental visit did not significantly differ between groups. A history of a breast cancer diagnosis did not increase the odds of gingivitis [odds ratio $(\mathrm{OR}): 1.32$; 95 percent confidence interval (CI): 0.53-3.63], periodontitis (OR: 1.82; 95 percent CI: $0.89-4.01$ ), or poor selfperceived oral health (OR: 0.89; 95 percent CI: 0.61-1.33) after adjusting for age, race, education, dental care utilization, and smoking status.

Conclusions: In this sample, a history of breast cancer does not significantly impact periodontal health, self-perceived oral health, and dental care utilization. However, efforts should be made to assure that breast cancer survivors have dental insurance.
\end{abstract}

\section{Introduction}

Breast cancer affects one in eight American women over the course of their lifetime. Breast cancer occurs more frequently in postmenopausal women than in premenopausal women; and the median age at diagnosis in the United States is 61 (1). For most women diagnosed with breast cancer, the 5-year survival rate is over 80 percent (1). The acute oral effects of chemotherapy and radiation include mucositis, xerostomia, caries, bleeding, and periodontal disease (2). However, long- term complications and late effects of cancer treatments on oral health are currently unexplored (3).

Postmenopausal breast cancer survivors are particularly vulnerable as their age places them at an increased risk for declining oral health in addition to experiencing skeletal complications of cancer therapy $(4,5)$. Research showed that chemotherapy and glucocorticoids, used to decrease the nausea associated with chemotherapy, accelerate skeletal bone loss (4) and that anti-estrogen therapy can be associated with bone loss (6). Systemic anti-estrogen use in 
postmenopausal women includes the use of tamoxifen and aromatase inhibitors (AIs) (7). AI use is associated with significant declines in bone mineral density (BMD) (8). Reduced estrogen levels such as those in postmenopausal women have been linked to the pathogenesis of periodontal disease (5). The fact that low BMD is cited as a risk factor for periodontitis suggests that cancer therapies may be a risk factor for periodontitis (9-11).

Understanding factors that can affect breast cancer survivors' quality of life is important (12). Oral pain and xerostomia have significant effects on reducing the oral health-related quality of life (OHRQoL) and functional capabilities of patients and, in some, nutritional status $(13,14)$. Moreover, self-perceived oral health has been shown to be a key determinant of dental care and overall health satisfaction (15).

Breast cancer survivors have been shown to receive fewer preventive services such as influenza vaccinations, cholesterol screening, and bone densitometry than age-matched controls due to the cancer diagnosis shifting attention away from noncancer routine medical services (16). Although understanding dental care utilization patterns in this population is critical for the assessment of oral health among breast cancer survivors, the utilization patterns of this population remain largely unknown.

As there is a lack of evidence concerning breast cancer survivors' oral health, the objectives of this population-based analysis were to determine the prevalence of periodontal diseases, the perceived oral health, and the patterns of dental care utilization in women ages 50-85 with and without a history of breast cancer in the United States.

\section{Methods}

\section{Database}

The Institutional Review Board at the University of Michigan declared this study to be exempt because National Health and Nutrition Surveys (NHANES) are publicly available and the data are de-identified. Data for this study were obtained from the NHANES 1999-2000, 2001-2002, 2003-2004 public datasets (17). The NHANES are designed to obtain information on the health and nutritional status of the noninstitutionalized population of the United States and are conducted by the National Center for Health Statistics. The sampling plan of each of the NHANES followed a highly stratified multistage probability design in which a sample was selected to provide national estimates. Methods for the standardized interviews, dental examinations, and procedures for human protection and consent have been described in detail elsewhere (17).

The starting sample for this analysis was 3,354 women 50-85 years who had participated in the examination part in the NHANES 1999-2004 and for whom valid questionnaire and cancer data were available. A total of 177 of these women identified themselves as breast cancer survivors; the rest of the women did not indicate that they were survivors of any kind of cancer $(n=3,177)$. Although the results presented in Tables 1 and 2 were based on data from the 3,354 women, an additional 266 participants were excluded from the periodontal estimates because they had not received a dental examination. In addition, 94 women had to be excluded because within their dental examinations, they had not received a periodontal examination. The results in Table 3 and the periodontal regression analyses models were therefore based on data from 2,994 females (164 breast cancer survivors and 2,830 women without any cancer).

\section{Breast cancer diagnosis and age of diagnosis}

The independent variable, self-reported breast cancer diagnosis, was determined from the health questionnaire section of the examination interview conducted at the mobile examination center (MEC). The questions were as follows: "Have you ever been told by a doctor or other health professional that you had cancer or a malignancy of any kind?" and "What kind of cancer was it?" Only those respondents who indicated they had breast cancer were eligible for inclusion. Participants who indicated any other type of cancer were ineligible. The respondent's age at the time of breast cancer diagnosis was also available.

\section{Outcome measures}

\section{Periodontal conditions}

The NHANES dental health examinations have been described previously $(18,19)$. Dental examinations were conducted by standardized examiners in the MEC. The periodontal status of individuals in NHANES was assessed using randomly assigned half-mouths (one upper and one lower quadrant) for each individual using a NIDCR periodontal probe. Periodontal examination data between the NHANES differed. The 1999-2000 protocol specified attachment loss, periodontal pockets assessments at two sites per tooth, and the gingival sweep was used to assess gingival bleeding at the quadrant level, whereas in the 2001-2002 and in the 20032004 surveys, periodontal pocket depth, clinical attachment loss (CAL), and periodontal bleeding were taken at three sites per tooth $(18,19)$. In this analysis, to increase comparability between NHANES protocols, only two sites (mid facial and mesial facial) were analyzed. Gingivitis was defined as the presence or absence of gingival bleeding in one or more quadrants or one or more sites. We defined moderate periodontal disease as two or more interproximal sites with $\geq 4 \mathrm{~mm}$ of CAL or two or more interproximal sites with a probing depth 
Table 1 Demographic and Clinical Characteristics of US Women Ages 50-85 NHANES Stratified by Breast Cancer Status, 1999-2004

\begin{tabular}{|c|c|c|c|c|c|c|c|}
\hline \multirow[b]{3}{*}{ Characteristic } & \multirow{2}{*}{\multicolumn{3}{|c|}{$\begin{array}{l}\text { Breast cancer } \\
n=177(5.5 \%)\end{array}$}} & \multirow{2}{*}{\multicolumn{4}{|c|}{$\begin{array}{c}\text { No cancer } \\
n=3,177(94.5 \%)\end{array}$}} \\
\hline & & & & & & & \\
\hline & Obs & $\%$ & SE & Obs & $\%$ & SE & $P$-value \\
\hline \multicolumn{8}{|l|}{ Age } \\
\hline $50-55$ years & 16 & 16.1 & 4.3 & 765 & 39.5 & 1.1 & \\
\hline $56-65$ years & 44 & 28.6 & 4.8 & 1,040 & 27.8 & 1.2 & \\
\hline $66-75$ years & 52 & 31.8 & 4.4 & 704 & 20.9 & 1.0 & \\
\hline $76-85$ years & 65 & 23.5 & 4.1 & 668 & 11.8 & 0.8 & $<0.001$ \\
\hline \multicolumn{8}{|l|}{ Ethnicity } \\
\hline White & 133 & 89.2 & 2.2 & 1,706 & 75.8 & 2.0 & \\
\hline Black & 21 & 5.9 & 1.3 & 571 & 10.3 & 1.0 & \\
\hline Hispanic \& Mexican American & 23 & 4.9 & 1.6 & 900 & 13.9 & 1.8 & $<0.001$ \\
\hline \multicolumn{8}{|l|}{ Education } \\
\hline Less than high school & 51 & 20.9 & 3.0 & 1,305 & 27.5 & 0.95 & \\
\hline High school diploma & 52 & 34.2 & 4.3 & 780 & 28.7 & 0.91 & \\
\hline More than high school & 74 & 44.9 & 4.2 & 1,066 & 43.8 & 1.22 & 0.47 \\
\hline \multicolumn{8}{|l|}{ Poverty income ratio* } \\
\hline $\mathrm{PIR}<=1.350$ & 35 & 19.3 & 3.4 & 864 & 23.4 & 1.3 & \\
\hline $\mathrm{PIR}>1.351 \& \mathrm{PIR}<=3.500$ & 63 & 44.2 & 5.0 & 116 & 38.1 & 1.7 & \\
\hline $\mathrm{PIR}>3.501$ & 50 & 36.5 & 5.1 & 779 & 38.5 & 1.5 & 0.43 \\
\hline \multicolumn{8}{|l|}{ Smoking status } \\
\hline Current & 17 & 12.7 & 2.5 & 394 & 14.2 & 0.88 & \\
\hline Past & 64 & 36.7 & 4.6 & 836 & 28.2 & 0.88 & \\
\hline Never & 96 & 50.6 & 4.2 & 1,933 & 57.6 & 1.2 & 0.12 \\
\hline \multicolumn{8}{|l|}{ Diagnosis of osteoporosis } \\
\hline Yes & 49 & 35.3 & 1.1 & 556 & 16.9 & 0.58 & \\
\hline No & 125 & 64.7 & 1.0 & 2,601 & 83.1 & 0.58 & $<0.001$ \\
\hline \multicolumn{8}{|l|}{ Diabetes } \\
\hline Yes & 34 & 16.3 & 3.2 & 2,644 & 12.8 & 0.78 & \\
\hline No & 143 & 83.7 & 3.2 & 528 & 87.2 & 0.78 & 0.24 \\
\hline \multicolumn{8}{|l|}{ Cancer characteristics } \\
\hline Mean age diagnosis ${ }^{\dagger}$ & 177 & 62.9 & & & NA & & \\
\hline Mean time since diagnosis ${ }^{\ddagger}$ & 170 & 9.9 & & & NA & & \\
\hline \multicolumn{8}{|l|}{ Dental characteristics } \\
\hline \multicolumn{8}{|l|}{ Has dental insurance } \\
\hline Yes & 62 & 41.4 & 4.8 & 1,378 & 51.9 & 1.6 & \\
\hline No & 110 & 58.6 & 4.8 & 1,506 & 48.1 & 1.6 & 0.04 \\
\hline \multicolumn{8}{|l|}{ Last dental visit } \\
\hline$<=12$ months & 107 & 66.0 & 4.9 & 1,644 & 52.5 & 1.6 & \\
\hline $12-35$ months & 29 & 16.8 & 3.8 & 572 & 18.2 & 0.75 & \\
\hline 36-59 months & 40 & 17.2 & 3.1 & 920 & 29.3 & 0.83 & 0.17 \\
\hline \multicolumn{8}{|l|}{ Reason for last dental visit } \\
\hline Checkup & 96 & 56.9 & 4.1 & 1,360 & 49.4 & 1.30 & \\
\hline Emergency & 44 & 23.5 & 4.2 & 1,134 & 32.5 & 1.07 & \\
\hline Scheduled & 33 & 19.6 & 3.5 & 629 & 18.1 & 0.71 & 0.14 \\
\hline
\end{tabular}

* Poverty income ratio is the ratio based on family income, family size, and number of children in the family, for families with two or fewer adults, and on the age of the adults in the household.

+ Mean age of cancer diagnosis (range 37-85 years; SD \pm 10.8 years).

₹ Mean time since diagnosis (range 0-35 years; SD \pm 7.1 years) \pm Not all variables add to column number due to nonresponse.

$\geq 5 \mathrm{~mm}$ (not on the same tooth) and severe periodontitis as two or more interproximal sites with CAL $\geq 6 \mathrm{~mm}$, not on the same tooth, and one or more interproximal sites with PD $\geq 5 \mathrm{~mm}$ following previously published reports (20).

\section{Perceived oral health}

Perceived oral health was assessed by asking the respondents to assess their oral health on a 4 -point scale $(1=$ poor, $2=$ fair, 
Table 2 Perception of Oral Health Responses of Respondents with and without a Cancer Diagnosis $(n=3,354)$

\begin{tabular}{|c|c|c|c|}
\hline $\begin{array}{l}\text { Perceived oral health } \\
\text { characteristic }\end{array}$ & $\begin{array}{l}\text { Breast cancer } \\
n=177 \%(\mathrm{SE})\end{array}$ & $\begin{array}{l}\text { No cancer } \\
n=3,177 \%(\mathrm{SE})\end{array}$ & $P$-value* \\
\hline \multicolumn{4}{|l|}{ Perception of oral health } \\
\hline Excellent & $30.6(4.7)$ & $24.9(1.3)$ & \\
\hline Good & $40.5(4.5)$ & $37.7(1.0)$ & \\
\hline Fair & $22.9(4.9)$ & $22.3(0.9)$ & \\
\hline Poor & $6.0(2.9)$ & $15.1(0.1)$ & $0.04^{+}$ \\
\hline \multicolumn{4}{|c|}{ Limited foods due to teeth/mouth? } \\
\hline Always & $2.1(2.0)$ & $3.9(0.6)$ & \\
\hline Often & $1.7(0.3)$ & $2.7(0.3)$ & \\
\hline Sometimes & $12.2(3.1)$ & $10.4(0.7)$ & \\
\hline Seldom & $6.5(1.6)$ & $9.6(1.0)$ & \\
\hline Never & $77.5(4.6)$ & $73.4(1.4)$ & 0.18 \\
\hline \multicolumn{4}{|c|}{ Amount of saliva in mouth } \\
\hline Too little & $9.3(2.8)$ & $8.4(0.5)$ & \\
\hline Too much & $6.3(1.9)$ & $4.8(0.5)$ & \\
\hline Did not notice & $84.4(3.4)$ & $86.8(0.8)$ & 0.71 \\
\hline \multicolumn{4}{|c|}{ Dry mouth when eating? } \\
\hline Yes & $6.2(2.2)$ & $5.2(0.5)$ & \\
\hline No & $93.8(2.3)$ & $94.8(0.4)$ & 0.62 \\
\hline
\end{tabular}

3 = good, $4=$ excellent). In addition, the respondents indicated how often their oral health limits the kind of food they ate ( 5 -point scale with $1=$ never, $2=$ seldom, $3=$ sometimes, $4=$ often, $5=$ always), and whether their mouth was dry when eating (yes/no). The last question was the perceived amount of saliva, which was measured as "too little," "too much," or "did not notice."

\section{Dental care utilization}

Dental care utilization was divided into a dental visit within the last 12 months, 13-35 months ago, and longer than 35 months ago. Participants who had visited the dentist were asked to indicate whether their visit had been for a check-up, an emergency, or a scheduled appointment. Dental insurance status was assessed as yes/no.

\section{Putative confounders}

Factors that have been shown to be associated with periodontal disease were evaluated for confounding and effect modification. Variables obtained from the face-to-face interview included age (50-55, 56-65, 66-75, 76-85 years). Race/ ethnicity was categorized as the following mutually exclusive categories - non-Hispanic white, Mexican American and other Hispanic and non-Hispanic black. Other race/ ethnicities were excluded from the analysis due to the small numbers of respondents. Education level was categorized as less than 12 years of education, 12 years of education, or greater than 12 years of education. Poverty income ratio (PIR) is the ratio of family income to poverty threshold for a given family size and composition. Guidelines are issued each year in the Bureau of the Census, Department of Commerce. For this analysis, PIR was categorized as (low: 0. 00-1. 350, medium: 1. 351-3.500, high: $\geq 3.501$ ) based on suggested NHANES analytical guidelines (21). Smoking status was obtained from two questions: Have you smoked at least 100 cigarettes in life? and (2) do you now smoke cigarettes? Smoking was defined as current, past, and never smoker. Selfreported osteoporosis diagnosis was reported as yes/no. Age of breast cancer diagnosis was a continuous variable, and length of time since diagnosis was categorized as $0-5$ years, 6-11 years, and 12 and more years.

Descriptive statistics were performed for all variables. Bivariate measures of unadjusted association between breast cancer diagnosis and periodontal outcomes (gingivitis/ periodontitis), dental utilization, and perceived oral health were assessed with the Pearson chi-square test and $t$-test. Given the small sample of breast cancer survivors in NHANES 1999-2004 data sets, adjustment for potentially confounding factors in multivariate models was purposely limited. Multiple logistic regression analysis utilizing the

Table 3 Periodontal Characteristics of Postmenopausal Women Aged 50-85 Years, by Breast Cancer Diagnosis $(n=2,994)$

\begin{tabular}{lccc}
\hline Periodontal measure & Breast cancer & No cancer & \\
& $n=164$ & $n=2,830$ & $P$-value \\
\hline & $\%(\mathrm{SE})^{\star}$ & $\%(\mathrm{SE})^{\star}$ & \\
Gingivitis & $48.1(1.2)$ & $44.2(3.1)$ & $0.12^{+}$ \\
Periodontitis - moderate & $16.5(3.0)$ & $12.4(0.98)$ & $0.39^{+}$ \\
Periodontitis- severe & $3.6(1.2)$ & $4.2(0.63)$ & $0.61^{\dagger}$ \\
Gingival bleeding & $4.1(0.13)$ & $3.5(0.08)$ & $0.73^{+}$ \\
Probing depths 4mm (\%) & $28.6(1.7)$ & $23.4(1.2)$ & $0.39^{+}$ \\
& Mean (SE) & Mean (SE) & $P$-value \\
Maximum attachment loss $(\mathrm{mm})^{\Uparrow}$ & $3.3(0.35)$ & $3.2(0.07)$ & $0.69^{\ddagger}$ \\
Mean attachment loss (mm) & $1.2(0.04)$ & $1.1(0.02)$ & $0.89^{\ddagger}$ \\
Maximum probing depth & $2.2(0.09)$ & $2.4(0.06)$ & $0.74^{\ddagger}$ \\
Mean probing depth & $2.5(0.35)$ & $2.4(0.11)$ & $0.33^{\ddagger}$ \\
Mean number of teeth & $15.0(1.0)$ & $17.1(0.34)$ & $0.07^{\S}$ \\
\hline
\end{tabular}

Periodontitis - moderate: $\geq 2$ or more interproximal sites with $\geq 4 \mathrm{~mm}$ clinical attachment loss (not on the same tooth) or $\geq 2$ interproximal sites with probing depth $a \geq 5 \mathrm{~mm}$ (not on the same tooth).

Periodontitis - severe: $\geq 2$ interproximal sites with clinical attachment loss of $\geq 6 \mathrm{~mm}$ (not on the same tooth) and $\geq 1$ interproximal sites with probing depth $\geq 5 \mathrm{~mm}$.

* SE: standard error of the mean.

+ Comparisons were carried out using chi-square test of association.

* Comparisons were carried out using the two sample $t$-test.

" $\mathrm{mm}=$ millimeter.

$\S$ Comparisons were carried out using a two-sample Wilcoxon rank-sum (Mann-Whitney) test. 
manual backward selection method was used to assess the relationship between breast cancer diagnosis and periodontal outcomes while controlling for other covariates. A threshold $P$-value of 0.20 for removal of a variable was used when building the model in a backward stepwise manner. However, all decisions for inclusion of variables were based on clinical and statistical significance. Potential interactions between breast cancer diagnosis and covariates were also examined using the Mantel-Haenszel chi-square test of association. No significant interactions were found for any models; therefore, only the main effects models are reported. All analyses were conducted using a software package STATA v. 13, (STATA Corporation, College Station, TX, USA) to adjust for complex sampling design and examination sampling weights and to provide adjusted variance estimations. Thus, the number of participants per category is unweighted, whereas all means, percentages, and ORs are weighted to reflect the target population and standard errors and 95 percent CIs are adjusted for sampling design.

As nearly 11 percent of women ages 50-85 did not receive a complete periodontal examination, multiple-imputation analysis (22) was used to examine whether results based on the complete-case analyses remained stable after imputation of missing values and to allow for greater statistical power to detect associations of interest between the analysis variables. Using IVEware software (Imputation and Variance Estimation Software, Institute for Social Research, Ann Arbor, MI, USA) 20 imputed datasets were generated and then averaged to generate a final overall dataset. The resulting associations behaved no differently than in the models without imputation, therefore only the complete case analysis was provided.

\section{Results}

Descriptive characteristics for the full sample of 3,354 US women ages 50 years and older stratified by breast cancer status are presented in Table 1. It is important to note that these same analyses were also conducted with data from the restricted sample of 2,994 women for whom periodontal data were available. However, the comparisons of the results based on the full sample and the results based on the restricted sample showed that the two sets of analyses did not differ in the outcomes of the group comparisons. This lack of differences concerning whether the two groups differed in the characteristics analyzed reflect that no major changes in the percentages of responses had been found when the data from the restricted sample were compared with the percentages of responses from the full sample.

Approximately, 5.5 percent of our sample reported a diagnosis of breast cancer. The average age at diagnosis was 62.9 years ( \pm 10.8 standard deviation) and the range was $37-85$ years. The average time since diagnosis was 9.9 years \pm 7.1 years; range 0 - 35 years.
Breast cancer survivors were more likely to be older than 66 years $(P<0.001)$, more likely to be Non-Hispanic white $(P<0.001)$, and had a higher prevalence of osteoporosis (35.3 percent versus 16.9 percent; $P<0.001$ ) as compared with unaffected women. The groups did not differ in their level of education, PIR, smoking status, or prevalence of diabetes. Breast cancer survivors were less likely to have dental insurance than women without a breast cancer diagnoses (38.7 percent versus 49.1 percent; $P=0.04$ ). However, the two groups did not differ in the percentages of women with a dental visit nor in the reasons for these visits.

Table 2 presents the results of bivariate analyses of the responses to the four OHRQoL questions. Women with a history of breast cancer diagnosis perceived their oral health more positively than women without a breast cancer diagnosis $(P=0.04)$. The respondent's perception as to the amount of saliva they reported to have in general and when eating did not differ according to cancer diagnosis.

Table 3 provides an overview of the periodontal parameters for women with and without breast cancer diagnosis. The prevalence of gingivitis of breast cancer survivors was 48.1 percent (nonbreast cancer respondents: 44.2 percent) and the prevalence of periodontitis was 16.5 percent (nonbreast cancer respondents: 12.4 percent). There were no significant differences in the periodontal pocket depths and attachment loss parameters of the two groups. A trend was found related to the mean number of teeth present with breast cancer survivors having on average 15.0 teeth present as compared with nonbreast cancer participants who have 17.1 teeth present $(P=0.07)$.

Multivariate analyses describing the periodontal health, perceived oral health, and dental utilization practices of survivors are shown in Table 4 . The periodontal health outcomes and oral health models were adjusted for age, race/ethnicity, education, dental visit within 12 months, and smoking. For the dental utilization model, as dental utilization was the outcome of interest, health insurance status was placed in the model as a covariate. As the prevalence of severe periodontal disease was low in both groups of women (see Table 3), no multivariate analysis with the dependent variable "severe periodontitis" was possible. Therefore, for the multivariate models, periodontitis was defined as including both moderate and severe periodontal disease.

Having had a breast cancer diagnosis was not associated with an increase in the odds of either gingivitis [odds ratio (OR): 1.24; 95 percent confidence interval (CI): 0.64, 2.53] or periodontitis (OR: 1.7; 95 percent CI: $0.96,3.02$ ) in either the unadjusted or adjusted models. Being nonwhite, smoking, and having a dental visit more than 12 months ago were associated with the odds of periodontitis after controlling for other covariates.

A breast cancer diagnosis was inversely associated with a woman's oral health perception (OR: 0.68; 95 percent 
Table 4 Weighted Estimates of Crude and Adjusted Odds Ratios and Designed-Based $95 \% \mathrm{Cl}$, of the Association of Cancer Diagnosis and Periodontal Measures, Perceived Oral Health, and Dental Visit within the Last 12 Months

\begin{tabular}{|c|c|c|c|c|c|c|c|c|}
\hline \multirow[b]{3}{*}{ Characteristics } & \multicolumn{8}{|c|}{ Dependent variable } \\
\hline & \multicolumn{2}{|c|}{ Gingivitis* } & \multicolumn{2}{|c|}{ Periodontitis $^{\dagger}$} & \multicolumn{2}{|c|}{$\begin{array}{l}\text { Perception of } \\
\text { oral health }{ }^{\ddagger}\end{array}$} & \multicolumn{2}{|c|}{ Dental utilization ${ }^{\S}$} \\
\hline & $\mathrm{OR}$ & $95 \% \mathrm{Cl}$ & OR & $95 \% \mathrm{Cl}$ & $\mathrm{OR}$ & $95 \% \mathrm{Cl}$ & OR & $95 \% \mathrm{Cl}$ \\
\hline \multicolumn{9}{|l|}{ Unadjusted estimates } \\
\hline Breast cancer diagnosis & 1.24 & $0.64-2.53$ & 1.7 & $0.96-3.02$ & 0.68 & $0.48-0.96$ & 0.80 & $0.53-1.24$ \\
\hline \multicolumn{9}{|l|}{ Adjusted estimates } \\
\hline Cancer diagnosis & 1.32 & $0.53-3.62$ & 1.82 & $0.89-4.01$ & 0.89 & $0.61-1.33$ & 0.73 & $0.43-1.21$ \\
\hline \multicolumn{9}{|l|}{ Age } \\
\hline $50-55$ years & 1.0 & & & & & & & \\
\hline $56-65$ years & 0.70 & $0.56-0.98$ & 1.0 & $0.59-1.7$ & 0.87 & $0.69-1.10$ & 1.3 & $0.97-1.90$ \\
\hline $66-75$ years & 0.76 & $0.55-1.23$ & 1.1 & $0.61-1.8$ & 0.78 & $0.61-1.04$ & 1.4 & $1.01-1.93$ \\
\hline $76-85$ years & 0.87 & $0.55-1.54$ & 0.73 & $0.43-1.3$ & 0.65 & $0.46-0.89$ & 2.0 & $1.54-2.85$ \\
\hline \multicolumn{9}{|l|}{ Ethnicity } \\
\hline White (Ref) & 1.0 & & 1.0 & & 1.0 & & 1.0 & \\
\hline Black & 1.13 & $0.66-1.72$ & 1.7 & $1.2-2.6$ & 1.9 & $1.52-2.45$ & 1.8 & $1.41-2.22$ \\
\hline Hispanic \& Mexican-American & 1.52 & $0.92-2.43$ & 2.0 & $1.3-3.1$ & 2.0 & $1.44-2.71$ & 1.4 & $0.94-2.04$ \\
\hline \multicolumn{9}{|l|}{ Education } \\
\hline$<$ High school (Ref) & 1.0 & & & & 1.0 & & & \\
\hline High school & 0.96 & $0.50-1.44$ & 0.82 & $0.50-1.4$ & 0.70 & $0.53-0.92$ & 0.51 & $0.36-0.72$ \\
\hline >High school & 1.01 & $0.63-1.42$ & 0.90 & $0.48-1.6$ & 0.52 & $0.40-0.66$ & 0.25 & $0.18-0.34$ \\
\hline \multicolumn{9}{|l|}{ Smoking status } \\
\hline Never & 1.0 & & 1.0 & & 1.0 & & 1.0 & \\
\hline Former & 1.07 & $0.61-1.70$ & 1.1 & $0.67-1.8$ & 1.1 & $0.83-1.34$ & 0.94 & $0.76-1.25$ \\
\hline Current & 1.09 & $0.64-1.52$ & 2.0 & $1.24-3.4$ & 1.8 & $1.32-2.35$ & 1.7 & $1.22-2.32$ \\
\hline \multicolumn{9}{|l|}{ Last dental visit } \\
\hline$>12$ months & 1.0 & & 1.0 & & 1.0 & & & \\
\hline$<=12$ months & 0.74 & $0.53-1.14$ & 0.47 & $0.44-0.68$ & 0.55 & $0.33-0.67$ & & Outcome \\
\hline \multicolumn{9}{|l|}{ Dental insurance } \\
\hline Yes & & & & & & & 0.73 & \\
\hline No & & & & & & & 1.0 & $0.59-0.92$ \\
\hline
\end{tabular}

* The gingivitis model was adjusted for age, race/ethnicity, education, having dental visit within 12 months, and smoking status $(n=2,994)$.

+ Periodontitis includes both moderate and severe cases. The periodontitis model was adjusted for age, race/ethnicity, education, smoking status, and having dental visit within past 12 months $(n=2,994)$.

\# Perception of oral health was assessed on a 4-point scale ranging from 1 = "poor" to 4 = "excellent oral health." Perception of oral health models were adjusted for age, race/ethnicity, education, dental visit within 12 months, and smoking status $(n=3,312)$.

$\S$ For the dental utilization regression model, as dental utilization is the outcome of interest, the model was adjusted for age, race/ethnicity, education, presence of dental insurance, and smoking status $(n=2,956)$.

CI: $0.48,0.96)$ in unadjusted analyses. However, the relationship between cancer diagnosis and perception of oral health was attenuated after adjusting other factors (OR: 0.89; 95 percent CI: $0.61,1.33$ ) (Table 4). Having lower educational attainment, being a current smoker, and being nonwhite were strongly associated with a higher probability of a lower perception of oral health.

A diagnosis of breast cancer was not associated with having a dental visit within the last year. Having higher educational attainment and being a current smoker and having dental insurance were significantly associated with a higher likelihood of having a dental visit within the last year.

\section{Discussion}

The aim of this study was to examine the relationship between a breast cancer diagnosis and periodontal diseases, perceived oral health, and dental care utilization using a large population based representative sample in the United States. This research is the first study that specifically investigated the oral health status of postmenopausal breast cancer survivors.

Prevalence estimates of breast cancer for women with breast cancer diagnoses in our study were slightly higher than those reported in two other studies that also used NHANES data to examine women diagnosed with breast cancer versus not having such a diagnosis $(23,24)$. Both of these studies 
found that approximately 4 percent of the women had been diagnosed with breast cancer, whereas we identified 5.5 percent of women with breast cancer diagnoses in our sample. However, this difference is most likely due to the fact that these two studies included data from women 35 years and older, whereas only data from women 50 years of age and older were included in our study. Similarly to our findings, these two publications also found that women with a diagnosis of breast cancer were significantly older than those with no history of cancer (64.5 years versus 53.3 years). In addition, the results from our study are also consistent with the findings from the two previous studies in finding an average time since diagnosis of nearly 10 years.

Approximately 66 percent of survivors reported a dental visit within the last year. This finding is consistent with the results of the 2000-2004 Medical Expenditure Panel Survey that also showed that 66 percent of breast cancer survivors had a dental visit within the past year (25). It is important in this context to note that breast cancer survivors in our sample were significantly less likely to report having dental insurance than women without a cancer diagnosis. This finding might be due to fact that the respondents in the breast cancer group were older than the women in the control group and thus may be less likely to have no dental insurance due to retirement or to not being employed.

However, the finding of women diagnosed with breast cancer being less likely to have dental insurance but nevertheless having a high utilization rate is inconsistent with findings in other areas of health care: Other studies showed that women with breast cancer diagnoses were less likely to have preventive care such influenza shots (16) than women without a cancer diagnosis. However, other authors examined the question of whether a breast cancer diagnosis modifies care-seeking behavior for comorbid conditions among older adults $(26,27)$. It has been speculated that once a woman becomes engaged in the healthcare system, they are likely to remain engaged (28). Future research should explore which factors determine preventive dental care utilization pattern among breast cancer survivors because good oral health is an important factor for the overall oral health of this population.

Interestingly, women who had a breast cancer diagnosis had a significantly better perception of their oral health than women without a cancer diagnosis in unadjusted analysis. However, the relationship was attenuated after controlling for confounding factors. Although we could not find any studies exploring the oral health perceptions of women with versus without a breast cancer diagnosis, a 5 year investigation of bisphosphonate zolendronic acid therapy in breast cancer patients assessed the women's OHRQoL at the completion of the study. The authors found no difference in the prevalence of impacts on oral health between patients with breast cancer diagnoses who had used versus not used zoledronate (29).
One explanation for our findings of no difference in perception of oral health could be that in a population with a cancer diagnosis, oral health problems play a secondary role in those with potentially life-threatening health problems (29). A feeling of mouth dryness while eating has been shown as a strong risk factor associated with poor OHRQOL (30). In our sample, the majority of cancer survivors indicated that their amount of saliva was "just right." As self-perceived oral health is shown to be a key determinant of food selection and dental care (31), further investigations into cancer treatments and OHRQoL are warranted.

Our results show no association between gingivitis or periodontitis and having had breast cancer among postmenopausal women. One possible explanation for this negative finding may be the consistent use of regular preventive dental services by breast cancer survivors. Another possibility for the lack of an association may be related to the predominant use of tamoxifen in this sample. Nearly 79 percent of women with a breast cancer diagnosis with a periodontal examination reported the use of tamoxifen. Before 2002, tamoxifen was the most prescribed antiestrogen drug for postmenopausal women with estrogen receptor-positive breast cancers (32). At present, no clinical data are available regarding the impact of tamoxifen and oral health. However, human gingival fibroblasts are a target tissue for sex hormones, and in vitro studies have demonstrated that tamoxifen may decrease the stimulatory effect of estrogen on human gingival fibroblast proliferation (33). Guidelines recommend that AIs are to be used at some point during the treatment of estrogen receptorpositive early-stage breast cancer in postmenopausal women (34). As AI use is associated with significant skeletal BMD loss (35) and oral bone correlates with skeletal BMD, women may be at risk for oral bone loss with the use of AIs $(36,37)$.

Limitations of this analysis include the cross-sectional design that prevents the determination of causality. Another potential limitation is healthy participation bias. Women who had received periodontal exams were more likely to be younger than 60 years of age, non-Hispanic white, married, have higher levels of education and family incomes, be nonsmokers, and report having dental insurance. Women were less likely to have a periodontal examination if they selfreported diabetes, osteoporosis, or cancer. Another limitation involves the representativeness of the breast cancer participants in NHANES for all women with a history of breast cancer. Also roughly 11 percent of women ages 50-85 did not have a complete periodontal examination severely limiting the robustness of the periodontal models. To address this, a multiple imputation technique was used for the missing periodontal variables. However, the resulting associations were essentially the same as in the models without imputation, leading us to believe that there is no association between 
diagnosis of breast cancer and periodontal diseases in this sample. Finally, the use of partial-mouth periodontal examination within the NHANES is recognized to underestimate periodontal disease prevalence resulting in disease misclassification thus attenuating the strength of the association between breast cancer diagnosis and periodontal conditions (38). Furthermore, the small number of breast cancer survivors coupled with the small number of periodontal examinations may have affected our ability to effectively capture the association between a breast cancer diagnosis and periodontal disease.

Another limitation of this analysis is the lack of information related to breast cancer treatments. The rationale and selection of breast cancer treatments is complex and based on many prognostic and predictive factors including tumor histology and grade, the clinical and pathologic stage, lymph node involvement, tumor hormone receptor content, tumor HER2 status, comorbid conditions, age, and patient preference. As such, there is likely great variation in the breast cancer treatments that these breast cancer survivors received. Also, as chemotherapy often accelerates skeletal bone loss, women counteract these effects by taking supplemental doses of calcium and vitamin D, which was not addressed in this analysis. Finally, The NHANES may disproportionately include long-term survivors (10 years or more) compared with the general population that includes women who have shorter survival periods.

Despite these limitations, a notable strength of this study is the diverse age and ethnicity of this national representative sample of US women. Of importance is the finding that breast cancer survivors are significantly less likely to have dental insurance compared to postmenopausal women without a cancer diagnosis. However, breast cancer survivors demonstrate regular preventive dental attendance that in turn may impact their overall perception of oral health.

Although a history of breast cancer was not associated with an increased prevalence of periodontal disease in this analysis, there is still a need for further examination of the impact of specific cancer treatment agents on the oral health and OHRQoL of postmenopausal breast cancer survivors. Prospective clinical studies assessing the specific role of breast cancer treatments, particularly chemotherapy and anti-estrogen therapies, are needed to elucidate the relationship between a breast cancer diagnosis and oral health status.

\section{Acknowledgments}

This work was supported by the University of Michigan Clinical Research Unit (NIH/CTSA UL1RR024986) and the National Institute of Dental \& Craniofacial Research
(NIDCR) grant 1K23DEO21779. We wish to thank Giselle Kolenic, University of Michigan, for her expert statistical guidance on the project and Anne Newman for her contribution to an earlier version of the manuscript. Drs. Taichman, Griggs and Inglehart report no conflicts of interest related to this study.

\section{References}

1. National Cancer Institute. Breast cancer statistics. 2013 [cited 2014 Oct 18]. Available from: http://www.cancer.gov/ cancertopics/types/breast

2. Dreizen S. Oral complications of cancer therapies. Description and incidence of oral complications. NCI Monogr. 1990;9:11-5.

3. Aziz NM. Late effects of cancer treatments: Surgery, radiation therapy, chemotherapy. In: Chang AE, Hayes DF, Kinsella T, Pass HI, Schiller JH, Stone R, Strecher V, editors. Oncology: An evidence based approach. New York: Springer-Verlag; 2006. p. 1768-90.

4. Khan MN, Khan AA. Cancer treatment-related bone loss: a review and synthesis of the literature. Curr Oncol. 2008; 15(Suppl 1):S30-40.

5. Wactawski-Wende J. Periodontal diseases and osteoporosis: association and mechanisms. Ann Periodontol. 2001;6(1): 197-208.

6. Van Poznak C, Sauter NP. Clinical management of osteoporosis in women with a history of breast carcinoma. Cancer. 2005;104(3):443-56.

7. Verma S, Jackisch C. Comparing guidelines for adjuvant endocrine therapy in postmenopausal women with breast cancer: a coming of age. Expert Rev Anticancer Ther. 2011; 11(2):277-86.

8. Gralow JR, Biermann JS, Farooki A, et al. NCCN Task Force Report: Bone Health in Cancer Care. J Natl Compr Canc Netw. 2009;7(Suppl 3):S1-32. quiz S33-S35.

9. Tezal M, Wactawski-Wende J, Grossi SG, Ho AW, Dunford R, Genco RJ. The relationship between bone mineral density and periodontitis in postmenopausal women. J Periodontol. 2000;71(9):1492-8.

10. Iwasaki M, Nakamura K, Yoshihara A, Miyazaki H. Change in bone mineral density and tooth loss in Japanese communitydwelling postmenopausal women: a 5-year cohort study. J Bone Miner Metab. 2012;30(4):447-53.

11. Genco RJ, Borgnakke WS. Risk factors for periodontal disease. Periodontol 2000. 2013;62:59-94.

12. Montazeri A. Health-related quality of life in breast cancer patients: a bibliographic review of the literature from 1974 to 2007. J Exp Clin Cancer Res. 2008;27:32-63.

13. Lee IC, Shieh TY, Yang YH, Tsai CC, Wang KH. Individuals' perception of oral health and its impact on the health-related quality of life. J Oral Rehabil. 2007;34(2):79-87.

14. Locker D. Dental status, xerostomia and the oral health-related quality of life of an elderly institutionalized population. Spec Care Dentist. 2003;23(3):86-93. 
15. Kressin N, Spiro A 3rd, Bosse R, Garcia R, Kazis L. Assessing oral health-related quality of life: findings from the normative aging study. Med Care. 1996;34(5):416-27.

16. Earle CC, Neville BA. Under use of necessary care among cancer survivors. Cancer. 2004;101(8):1712-9.

17. Centers for Disease Control and Prevention (CDC). National Center for Health Statistics (NCHS). National Health and Nutrition Examination Protocol. Hyattsville, MD: U.S. Department of Health and Human Services, Centers for Disease Control and Prevention. 2014 [cited 2014 Oct 15]. Available from: http://www.cdc.gov/nchs/nhanes/ nhanes_questionnaires.htm.

18. Dye BA, Barker LK, Selwitz RH, et al. Overview and quality assurance for the National Health and Nutrition Examination Survey (NHANES) oral health component, 1999-2002. Community Dent Oral Epidemiol. 2007;35(2):140-51.

19. Dye BA, Nowjack-Raymer R, Barker LK, et al. Overview and quality assurance for the oral health component of the National Health and Nutrition Examination Survey (NHANES), 2003-04. J Public Health Dent. 2008;68(4): 218-26.

20. Page RC, Eke PI. Case definitions for use in population-based surveillance of periodontitis. J Periodontol. 2007;78(7 Suppl): 1387-99.

21. U.S. Department of Health and Human Services US. Analytic and Reporting Guidelines: The Third National Health and Nutrition Examination Survey, NHANES III (1988-94). 1996. 2009 [cited 2014 Jun 20]. Available from: http://www.cdc.gov/ nchs/data/nhanes/nhanes3/nh3gui.pdf.

22. Raghunathan TE, Lepkowski JM, van Hoewyk M, Solenberger PW. A multivariate technique for multiply imputing missing values using a sequence of regression models. Survey Methodol. 2001;27:85-95.

23. Parente V, Hale L, Palermo T. Association between breast cancer and allostatic load by race: National Health and Nutrition Examination Survey 1999-2008. Psychooncology. 2013;22:621-8.

24. Milliron BJ, Vitolins MZ, Tooze JA. Usual dietary intake among female breast cancer survivors is not significantly different from women with no cancer history: results of the National Health and Nutrition Examination Survey, 2003-2006. J Acad Nutr Diet. 2014;114:932-7.

25. Findley PA, Sambamoorthi U. Preventive health services and lifestyle practices in cancer survivors: a population health investigation. J Cancer Surviv. 2009;3(1):43-58.

26. Earle CC, Burstein HJ, Winer EP, Weeks JC. Quality of non-breast cancer health maintenance among elderly breast cancer survivors. J Clin Oncol. 2003;21(8):1447-51.

27. Duffy CM, Clark MA, Allworth JE. Health maintenance and screening in breast cancer survivors in the United States. Cancer Detect Prev. 2006;30(1):52-7.
28. Hanchate AD, Clough-Gorr KM, Ash AS, Thwin SS, Silliman RA. Longitudinal patterns in survival, comorbidity, healthcare utilization and quality of care among older women following breast cancer diagnosis. J Gen Intern Med. 2010;25(10):1045-50.

29. Rathburn J, et al. Osteonecrosis of the jaw and oral health-related quality of life after adjuvant zoledronic acid: an adjuvant zoledronic acid to reduce recurrence trial subprotocol (BIG01/04). J Clin Oncol. 2013;31:2685-91.

30. Kotzer RD, Lawrence HP, Clovis JB, Matthews DC. Oral health-related quality of life in an aging Canadian population. Health Qual Life Outcomes. 2012;10:50-81. [cited 2013 Jan 14]. Available from: http://www.ncbi.nlm.nih.gov/ PMC/articles/PMC3480903.

31. Seirawan H, Sundaresan S, Mulligan R. Oral health-related quality of life and perceived dental needs in the United States. J Public Health Dent. 2011;71(3):194-201.

32. Locker D, Matear D, Stephens M, Jokovic A. Oral health-related quality of life of a population of medically compromised elderly people. Community Dent Health. 2002;19(2):90-7.

33. Baum M, Budzar AU, Cuzick J, et al. Anastrozole alone or in combination with tamoxifen versus tamoxifen alone for adjuvant treatment of postmenopausal women with early breast cancer: first results of the ATAC randomised trial. Lancet. 2002;359(9324):2131-9.

34. Soory M, Tilakaratne A. Modulation of androgen metabolism by phenytoin, oestradiol and tamoxifen in human gingival fibroblasts. J Clin Periodontol. 2003;30(6): 556-61.

35. Burstein HJ, Prestrud AA, Seidenfeld J, et al. American Society of Clinical Oncology clinical practice guideline: update on adjuvant endocrine therapy for women with hormone receptor-positive breast cancer. J Clin Oncol. 2010;28(23):3784-96.

36. Coates AS, Keshaviah A, Thurlimann B, et al. Five years of letrozole compared with tamoxifen as initial adjuvant therapy for postmenopausal women with endocrine-responsive early breast cancer: update of study BIG 1-98. J Clin Oncol. 2007;25(5):486-92.

37. Makker A, Singh MM, Mishra G, Singh BP, Jain GK, Jadhav S. Relationship between bone turnover biomarkers, mandibular bone mineral density, and systemic skeletal bone mineral density in premenopausal and postmenopausal Indian women. Menopause. 2012;19(6):642-9.

38. Eke PI, Thornton-Evans GO, Wei L, Borgnakke WS, Dye BA. Accuracy of NHANES periodontal examination protocols. J Dent Res. 2010;89(11):1208-13. 